\title{
LncRNA LUADT1 sponges miR-195 to prevent cardiac endothelial cell apoptosis in sepsis
}

Zhimin Zhang ${ }^{1 \dagger}$, Mingzhu Lv ${ }^{3 \dagger}$, Xiang Wang ${ }^{1}$, Zheng Zhao ${ }^{2}$, Daolong Jiang ${ }^{2}$ and Lihua Wang ${ }^{2^{*}}$ (D)

\begin{abstract}
Background: The oncogenic role of the newly identified IncRNA LUADT1 has been revealed in lung adenocarcinoma. It was reported that LUADT1 plays a critical role in multiple human diseases. This study was carried out to investigate the role of LUADT1 in sepsis.

Methods: Sixty patients with sepsis and sixty healthy volunteers were recruited for this study. Plasma samples were collected from all participants. Human primary coronary artery endothelial cells were also used in this study. The expression of Pim-1, miR-195 and LUADT1 were detected by RT-qPCR. The interaction between miR-195 and LUADT1 was determined by overexpression experiments and luciferase activity assay. Cell apoptosis was detected by flow cytometry. The expression of apoptosis-related protein was detected by Western blotting.

Results: Bioinformatics analysis revealed the potential interaction between LUADT1 and miR-195, which was confirmed by dual luciferase reporter assay. LUADT1 was downregulated in patients with sepsis. Moreover, LPS treatment downregulated the expression of LUADT1 in primary cardiac endothelial cells. Overexpression of LUADT1 and miR195 did not affect the expression of each other in primary cardiac endothelial cells. Interestingly, overexpression of LUADT1 was found to upregulate the expression of Pim-1, a target of miR-195. In addition, it was found that overexpression of LUADT1 and Pim-1 reduced the enhancement effects of miR-195 on LPS-induced cardiac endothelial cell apoptosis.
\end{abstract}

Conclusion: In summary, LUADT1 may protect cardiac endothelial cells against apoptosis in sepsis by regulating the miR-195/Pim-1 axis.

Keywords: Sepsis, LUADT1, miR-195, Cardiac endothelial cells, Pim-1, Apoptosis

\section{Introduction}

Sepsis is a common and lethal clinical syndrome that occurs when severe systemic responses of body to infection, resulting in injury to its own tissues and organs (Cohen et al. 2015). Sepsis mostly affects elderly and patients complicated with other severe diseases, such

\footnotetext{
*Correspondence: yr2064@163.com

'Zhimin Zhang and Mingzhu Lv contributed equally to this work

2 Department of Clinical Laboratory, Affliated Dongfeng Hospital, Hubei University of Medicine, Shiyan 442008, Hubei, People's Republic of China Full list of author information is available at the end of the article
}

as cancer (Gotts and Matthay 2016). The development of sepsis causes the dysfunction of multiple organs and induces critical illness characterized by severe immune dysfunction and catabolism (Gotts and Matthay 2016). Multiple therapeutic approaches have shown promising efficacy in improving the conditions of sepsis animal model, and some approaches are under clinical validation (Hotchkiss and Karl 2003; Riedemann et al. 2003; Cronin et al. 1995). However, antibiotics, which are correlated with the high prevalence of resistance, are still the most commonly used drugs in the clinical treatment of sepsis (Liu et al. (2017)). original author(s) and the source, provide a link to the Creative Commons licence, and indicate if changes were made. The images or other third party material in this article are included in the article's Creative Commons licence, unless indicated otherwise in a credit line to the material. If material is not included in the article's Creative Commons licence and your intended use is not permitted by statutory regulation or exceeds the permitted use, you will need to obtain permission directly from the copyright holder. To view a copy of this licence, visit http://creativecommons.org/licenses/by/4.0/. 
The identification of genetic factors involved in the development and progression of sepsis provides novel insights into the development of targeted therapies (Giamarellos-Bourboulis and Opal 2016; Flores 2015; Poll et al. 2017). Besides protein-coding genes, the development and progression of sepsis is also accompanied with changes in the expression of non-coding RNAs (ncRNAs), such as miRNAs (Vasilescu et al. 2017). For instance, in mice sepsis model, miR-195 is upregulated, and inhibition of miR-195 can reduce the injuries of multiple organs (Zheng et al. 2015), suggesting that downregulation of miR-195 may be a potential therapeutic target for sepsis. LUADT1 is a newly identified oncogenic gene long non-coding RNA (lncRNA) in lung adenocarcinoma (Qiu et al. 2015). Our bioinformatics analysis revealed the potential interaction between LUADT1 and miR-195. This study was therefore carried out to investigate the potential interaction between LUADT1 and miR-195 in sepsis.

\section{Materials and methods Research subjects}

This study passed the revised board of the Affiliated Dongfeng Hospital, Hubei University of Medicine (DFLS-2019-007). Research subjects of the present study included 60 patients with sepsis and 60 healthy controls who were enrolled in aforementioned hospital between January and June of 2019, and their basic characteristics were shown in Table 1 . Inclusion criteria for sepsis patients: (1) Sepsis confirmed according to American College of Chest Physicians/Society of Critical Care Medicine criteria; (2) Sepsis patients with at least two of the followings when arrival ER and treated $>6 \mathrm{~h}, \mathrm{BT}>38{ }^{\circ} \mathrm{C}$ or $<36{ }^{\circ} \mathrm{C}, \mathrm{HR}>90 / \mathrm{min}, \mathrm{RR}>20 /$ min or $\mathrm{PaCO}_{2}<32 \mathrm{mmHg}$, WBC count $>12 \times 10^{9} / \mathrm{L}$ or $<4 \times 10^{9} / \mathrm{L}$ or immature form $>10 \%$. Age and sex matched healthy volunteers were recruited as healthy controls, and people with the following situations were excluded: systematic inflammatory disease, solid cancer or hematological malignancies, severe kidney or hepatic dysfunction, undergoing steroid or immunosuppressive therapy. Written form of the informed consent was signed by both patients and healthy controls.

\section{Plasma and cardiac endothelial cells}

All patients and healthy controls were subjected to blood extraction $(5 \mathrm{ml})$ under fasting conditions. Blood samples were transferred to EDTA tubes, followed by centrifugation at $1,200 \mathrm{~g}$ at room temperature for $10 \mathrm{~min}$ to prepare plasma samples. Human primary coronary artery endothelial cells (HCAECs, ATCC ${ }^{\circledR}$ PCS-100-020 ${ }^{\mathrm{TM}}$ ) were purchased from ATCC. Cells were cultured in Endothelial Cell Growth Medium MV containing 10\% FBS. Cells culture conditions were: $37{ }^{\circ} \mathrm{C}, 5 \% \mathrm{CO}_{2}$ and $95 \%$ humidity. Cells were harvested at $75-85 \%$ confluence to perform the following experiments.

\section{Transient transfections}

PcDNA3.1 vector expressing the full length of LUADT1 and Pim-1 cDNAs were constructed by GeneCopoeia (Guangzhou, China). Negative control (NC) miRNA, miR-195 mimic, antagomir and siPim-1 were synthesized by Sangon (Shanghai, China). Lipofectamine 2000 (Thermo Fisher Scientific) was used to transfect $10 \mathrm{nM}$ vector or $50 \mathrm{nM}$ miRNA into $10^{6}$ cells. Control (C) cells were un-transfected cells, and NC cells were transfected

Table 1 Basic characteristics of sepsis patients and healthy controls

\begin{tabular}{|c|c|c|c|}
\hline Characteristics & sepsis patients $(n=60)$ & Healthy controls $(n=60)$ & $P$ value \\
\hline Age (year) & $53.12 \pm 6.9$ & $54.37 \pm 6.7$ & 0.32 \\
\hline \multicolumn{4}{|l|}{ Gender (n/\%) } \\
\hline Male & $41(68.3 \%)$ & $41(68.3 \%)$ & \\
\hline Female & $19(31.7 \%)$ & $19(31.7 \%)$ & \\
\hline $\mathrm{BMI}\left(\mathrm{Kg} / \mathrm{m}^{2}\right)$ & $24.30 \pm 3.23$ & $23.54 \pm 2.91$ & 0.18 \\
\hline Albumin (g/L) & $25.761(22.346-34.975)$ & NA & \\
\hline WBC $\left(\times 10^{9} / L\right)$ & $13.017(4.129-28.438)$ & NA & \\
\hline APACHE II score & $19.6 \pm 5.1$ & NA & \\
\hline \multicolumn{4}{|l|}{ Source of sepsis (n/\%) } \\
\hline Respiratory tract infection & $21(35.0 \%)$ & NA & \\
\hline Urinary tract infection & $16(26.7 \%)$ & NA & \\
\hline Abdominal infection & $9(15.0)$ & NA & \\
\hline Pneumonia & $6(10.0 \%)$ & NA & \\
\hline Wound infection & $5(8.3 \%)$ & NA & \\
\hline Others & $3(5.0 \%)$ & NA & \\
\hline
\end{tabular}


with empty vector or NC miRNA. Cells were harvested at $24 \mathrm{~h}$ post-transfection.

\section{Dual luciferase reporter assay}

PGL3 Luciferase Reporter Vector (Promega) was used to construct LUADT1 vector (full length cDNA). Co-transfection of LUADT1 vector and miRNA NC or miR-195 mimic was performed through aforementioned methods. Dual-luciferase reporter assay kit (Promega) was used to measure the relative luciferase activity. Firefly luciferase activity was normalized to renilla luciferase.

\section{RNA preparation and qPCR}

Total RNAs were extracted from both tissue samples and in vitro cultured cells using Trizol reagent (Invitrogen). For LPS treatment, HCAECs were cultured in cell culture medium containing 0, 100,150, 200 and $500 \mathrm{ng} /$ $\mathrm{ml}$ LPS for $24 \mathrm{~h}$ before use. DNA eraser (Takara) was used to digest all RNA samples to remove genomic DNA. Reverse transcriptions (RTs) were performed using PrimeScript RT-polymerase (Takara) with total RNA samples as template. With cDNA samples as template, qPCR reaction mixtures were prepared using QuantiTect SYBR Green PCR Kit (QIAGEN). The expression levels of LUADT1 and Pim-1 mRNA were measured with GAPDH as endogenous control. Extraction of miRNAs from aforementioned samples and cells was performed using miRNeasy Mini Kit (QIAGEN). The expression levels of mature miR-195 were measured using All-inOne $^{\mathrm{TM}}$ miRNA qRT-PCR Detection Kit (Genecopoeia) with U6 as endogenous control. All PCR reactions were performed in triplicate manner and fold changes of gene expression levels were calculated using $2^{-\Delta \Delta C t}$ method.

\section{Western blot}

Cells were collected at $24 \mathrm{~h}$ post-transfection and washed twice in PBS. Cells were then lysed in RIPA solution containing protease inhibitor (Sigma-Aldrich) to prepare cell lysates. BCA assay kit (Sigma-Aldrich) was used to measure protein concentrations. Protein samples were denatured in boiling water for $10 \mathrm{~min}$. After that, equivalent amount of protein samples were separated by $10 \%$ SDSPAGE gel, followed by gel transfer to PVDF membranes. Membranes were subsequently blocked in PBS containing 5\% non-fat milk at room temperature for $2 \mathrm{~h}$. Then membranes were incubated with primary antibodies of rabbit anti-GAPDH (ab9845, Abcam) and anti-Pim-1 (ab98004, Abcam), caspase3 (ab13847, Abcam) at $4{ }^{\circ} \mathrm{C}$ for $12 \mathrm{~h}$. After that, membranes were further incubated with HRP Goat Anti-Rabbit (IgG) secondary antibody (ab6721, Abcam) at $25^{\circ} \mathrm{C}$ for $2 \mathrm{~h}$. ECL chemiluminescence kit (Pierce) was used to produce signals, which were normalized to GAPDH endogenous control using Image J v1.48 software.

\section{Flow cytometric analysis}

Cells were harvested at $24 \mathrm{~h}$ post-transfection and cell cultures were performed using 6-cm tissue culture dishes with cell culture medium supplemented $150 \mathrm{ng} / \mathrm{ml}$ LPS for another $24 \mathrm{~h}$. Pre-cold PBS was used to resuspend adherent cells. Alexa Fluor ${ }^{\circledR}$ 647/7-AAD apoptosis kit (BioLegend) was used to process cells with all steps performed following the manufacturer's instructions. Flow cytometry (Becton-Dickinson) was performed to detect apoptotic cells.

\section{Statistical analysis}

Data from 3 independent biological replicates were used to calculate the mean values, which were subjected to statistical analysis using SPSS software (version 18.0). Unpaired t test was used to explore the differences of two groups. Differences among multiple groups were analyzed by ANOVA (one-way) and Tukey test. $P<0.05$ was considered as statistically significant. Correlations were performed using Pearson's correlation coefficient.

\section{Results \\ LUADT1 was downregulated in patients with sepsis}

The expression of LUADT1 was detected in plasma from both sepsis patients $(n=60)$ and healthy controls $(n=60)$. It was observed that the expression levels of LUADT1 were significantly lower in sepsis patients then that in the control group (Fig. 1a, p< 0.05). Correlations between LUADT1 and miR-195 were analyzed by Pearson' correlation coefficient. The results showed that the expression levels of LUADT1 were significantly and inversely correlated with the expression levels of miR-195 in sepsis patients (Fig. $1 \mathrm{~b}, \mathrm{R}^{2}=0.0699, p<0.05$ ), while the expression levels of Pim-1 were positively correlated with that of LUADT1 (Fig. $1 \mathrm{c}, \mathrm{R}^{2}=0.1412, p<0.05$ ).

\section{LUADT1 and miR-195 can directly interact with each other} The potential interaction between LUADT1 and miR-195 was analyzed by the online program IntaRNA (https:// rna.informatik.uni-freiburg.de/IntaRNA/Input.jsp) for RNA-RNA interaction. It was observed that LUADT1 and miR-195 could form strong base paring between each other (Fig. 2a). Dual luciferase reporter assay was performed to further analyze the interaction between them. Compared with HCAECs co-transfected with LUADT1 vector and miRNA NC (NC group), HCAECs co-transfected with LUADT1 vector and miR-195 mimic (miR-195 group) showed significantly lower relative luciferase activity (Fig. 2b, p < 0.05). 

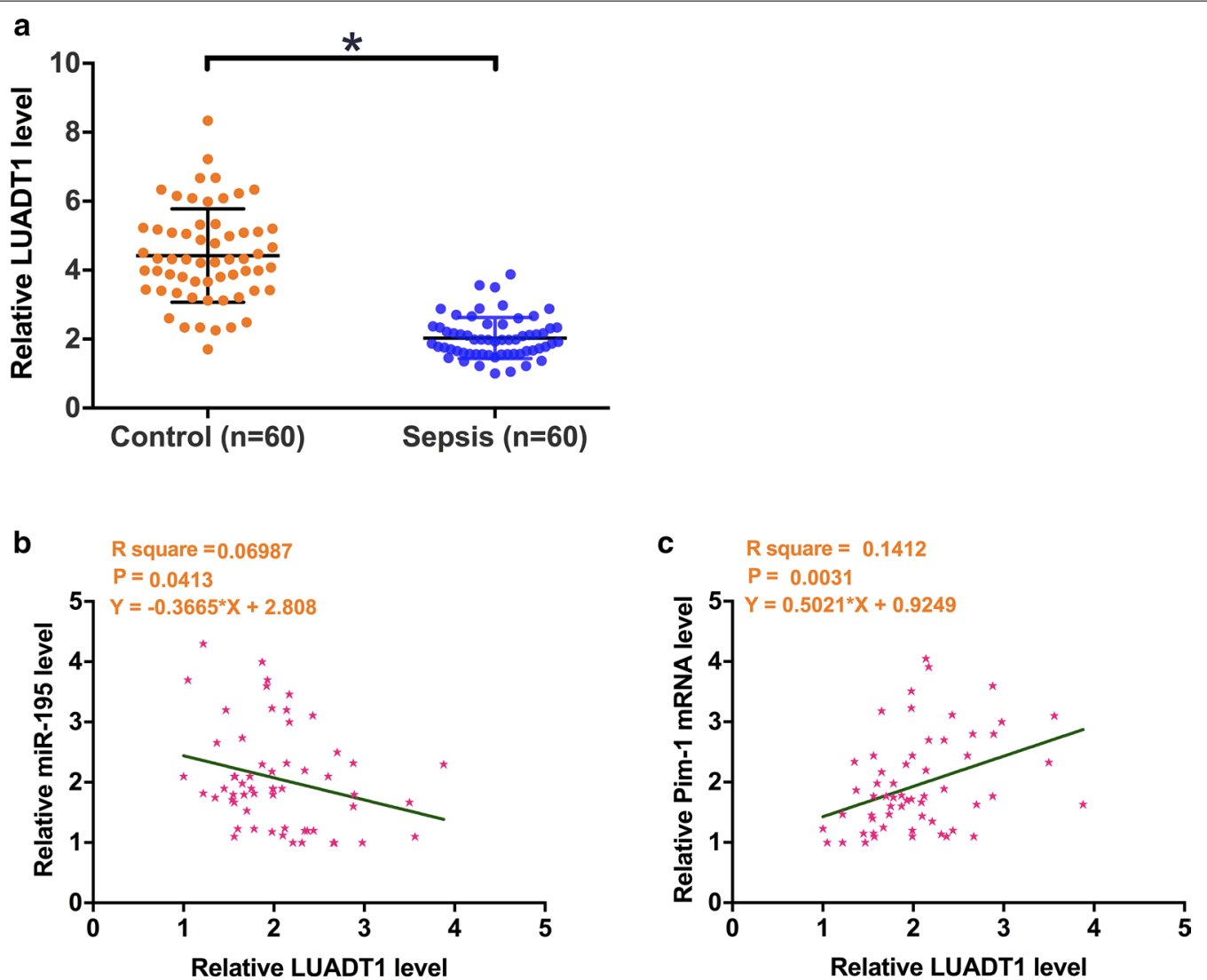

Fig. 1 LUADT1 was downregulated in patients with sepsis. The expression of LUADT1 was analyzed by measuring the levels of LUADT1 in plasma from both sepsis patients $(n=60)$ and healthy controls $(n=60)$. Data were compared between two groups by unpaired test. PCR reactions were repeated 3 times and mean values were presented (a). The mRNA expression levels of miR-195 and Pim-1 from sepsis patients were also measured by performing RT-qPCR. Correlations between LUADT1 and miR-195 (b)/Pim-1 mRNA (c) were analyzed by Pearson's correlation coefficient. Mean values were used in analyses. ${ }^{*}, p<0.05$
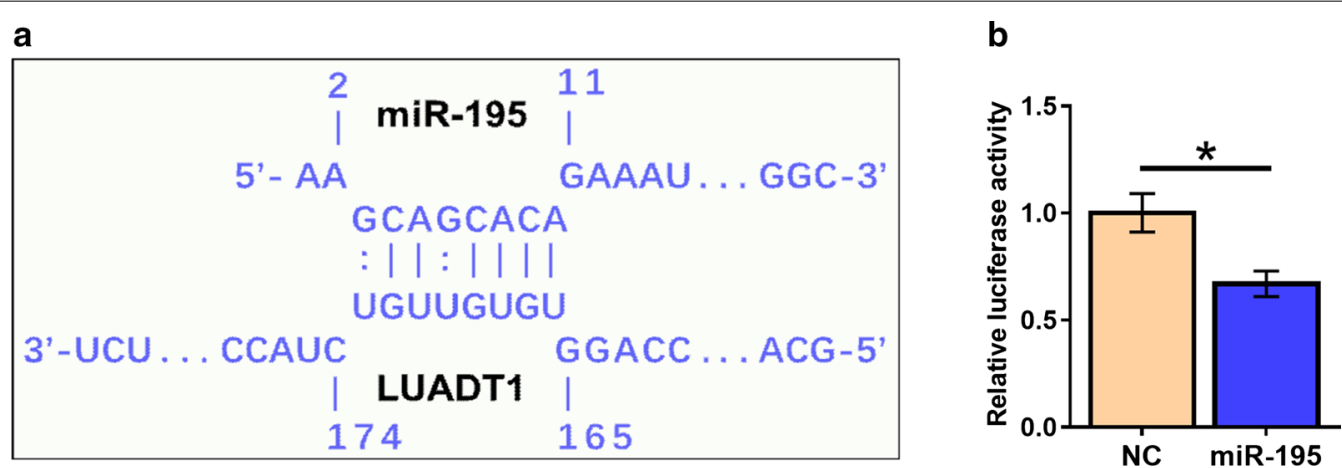

Fig. 2 LUADT1 and miR-195 can directly interact with each other. The potential interaction between LUADT1 and miR-195 was analyzed by IntaRNA (https://rna.informatik.uni-freiburg.de/IntaRNA/Input.jsp) (a). Dual luciferase assay was performed by co-transfecting LUADT1 vector and miRNA NC (NC group) or LUADT1 vector and miR-195 mimic (miR-195 group) into HCAECs. Experiments were repeated 3 times and mean values were presented (b). ${ }^{*}, p<0.05$ 


\section{Overexpression of LUADT1 and miR-195 did not affect} the expression of each other

To further analyze the interaction between LUADT1 and miR-195, HCAECs were transfected with LUADT1 expression vector and miR-195 mimic. Overexpression of LUADT1 and miR-195 were confirmed by RT-qPCR at $24 \mathrm{~h}$ post-transfection (Fig. $3 \mathrm{a}, \mathrm{p}<0.05$ ). Compared with the two control groups $\mathrm{C}$ and $\mathrm{NC}$, overexpression of LUADT1 and miR-195 did not significantly alter the expression of each other (Fig. 3b, p<0.05). RT-qPCR and Western blotting were performed to elucidate the effects of overexpression of LUADT1 and miR-195 on the expression of Pim-1, a downstream target of miR-195, at both mRNA and protein levels. Compared with $\mathrm{C}$ (un-transfected cells) and NC (empty pcDNA3.1 vector or NC miRNA transfection) groups, overexpression of LUADT1 upregulated, while overexpression of miR-195 downregulated Pim-1 expression at both mRNA (Fig. 3c, $\mathrm{p}<0.05)$ and protein (Fig. 3d, $\mathrm{p}<0.05)$ levels. Moreover, overexpression of miR-195 attenuated the effects of LUADT1 overexpression $(p<0.05)$.

\section{LUADT1 suppressed LPS-induced apoptosis of HCAECs} through miR-195/Pim-1

HCAECs were cultured in cell culture medium containing $0,100,150,200$ and $500 \mathrm{ng} / \mathrm{ml}$ LPS for $24 \mathrm{~h}$, followed
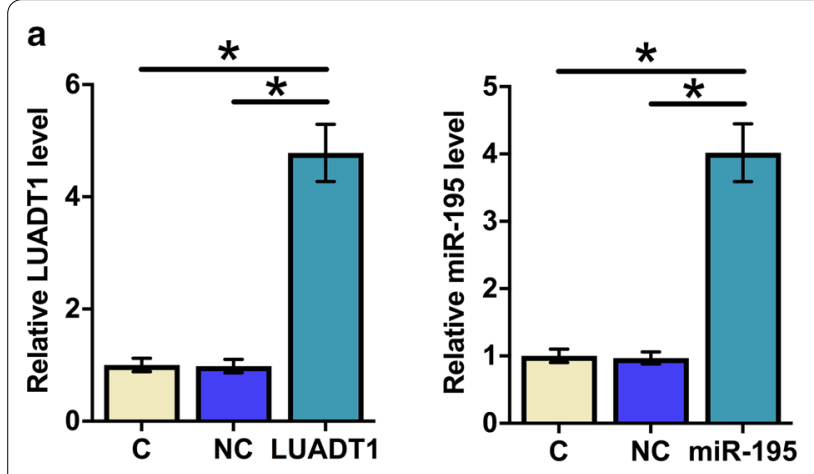

C

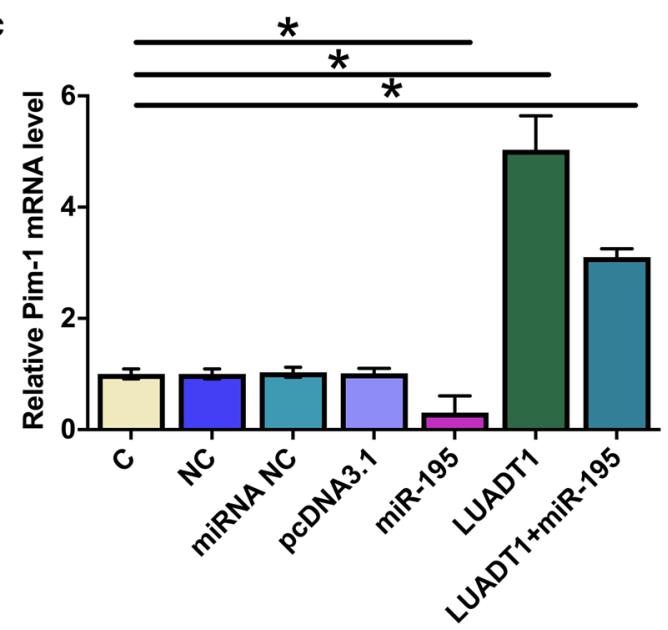

b
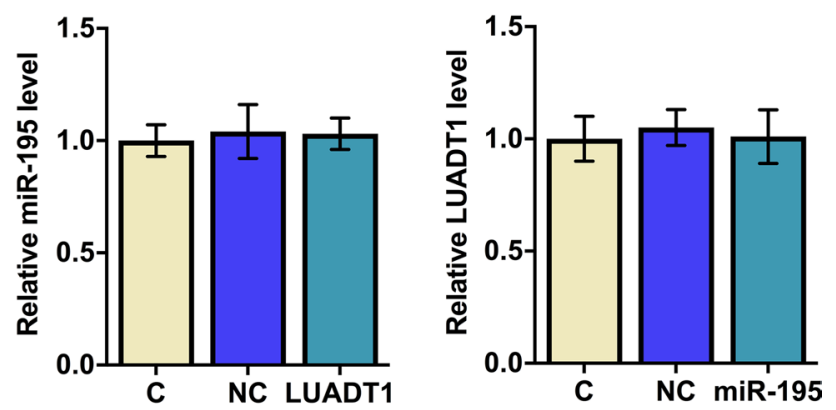

d

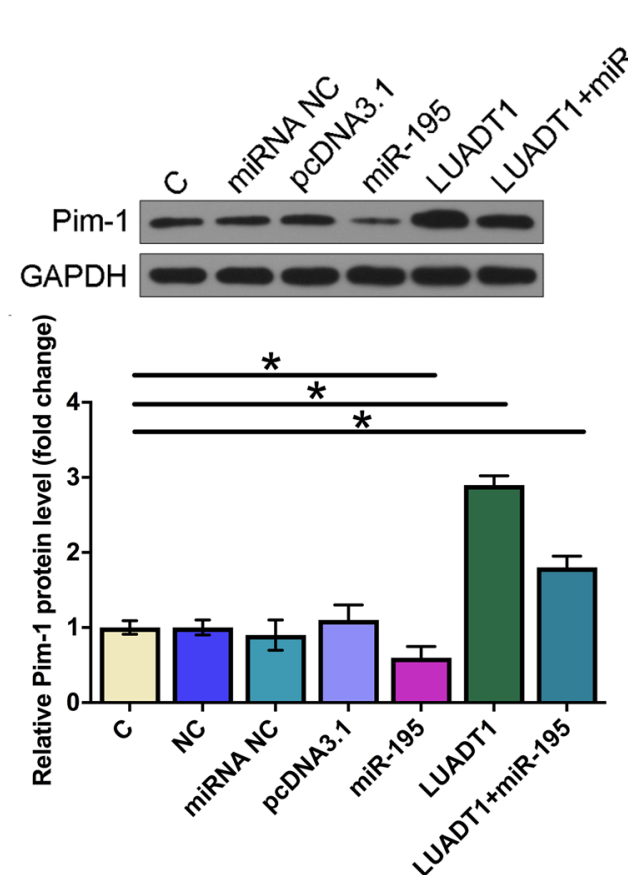

Fig. 3 Overexpression of LUADT1 and miR-195 did not affect the expression of each other. HCAECs were transfected with LUADT1 expression vector and miR-195 mimic. LUADT1 and miR-195 overexpression was confirmed by RT-qPCR at $24 \mathrm{~h}$ post-transfection (a). The effects of LUADT1 and miR-195 overexpression on the expression of each other were also analyzed by RT-qPCR at $24 \mathrm{~h}$ post-transfection (b). The effects of overexpression of LUADT1 and miR-195 mimic on the expression of Pim-1, a downstream target of miR-195, at both mRNA and protein levels were analyzed by RT-qPCR (c) and western blot (d). Experiments were repeated 3 times, and data were expressed a mean value. *, $p<0.05$ 
by measuring the expression levels of LUADT1 by qPCR. It was observed that LPS treatment downregulated the expression of LUADT1 in a dose-dependent manner (Fig. 4a, p <0.05). Cell apoptosis assay was performed to analyze the effects of LUADT1, miR-195 and Pim-1 overexpression on the apoptosis of HCAECs treated by $150 \mathrm{ng} / \mathrm{ml}$ LPS. Compared with C group and NC groups, cell apoptosis analysis showed that overexpression of LUADT1 and Pim-1 decreased, while overexpression of miR-195 increased the apoptotic rate of HCAECs. In addition, overexpression of miR-195 attenuated the effects of LUADT1 or Pim-1 overexpression (Fig. 4b, $\mathrm{p}<0.05)$. Caspase 3 activity was consistent with the apoptosis results (Fig. 4c). Cell apoptosis was detected after the overexpression of Pim-ko and LUADT1, knock-down of Pim-1 decreased the HCAEC apoptosis (Fig. 4d). The expression of Pim-1 and the cell apoptosis were examined after adding miR-195 antagomir at $150 \mathrm{ng} / \mathrm{mL}$ LPS. It showed that the expression levels of Pim-1 were decreased after LPS treatment, while increased after miR-195 antagomir treatment (Fig. 4e).

\section{Discussion}

The function of LUADT1 has only been investigated in lung adenocarcinoma (Qiu et al. 2015). It was observed that LUADT1 downregulated P27 by epigenetic pathway, thereby upregulating and promoting the proliferation of lung adenocarcinoma cells (Qiu et al. 2015). Our

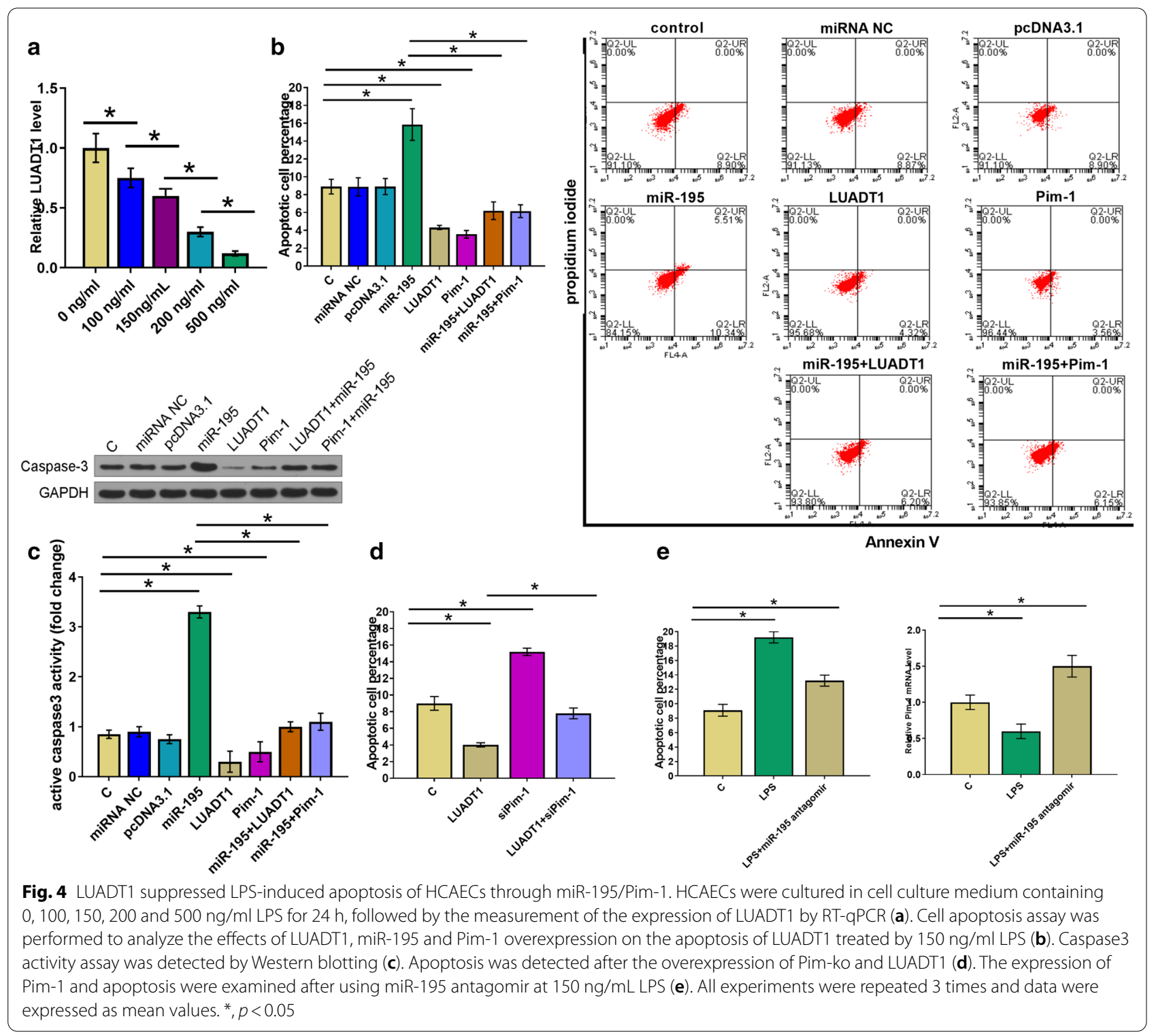


study revealed a new role of LUADT1 in sepsis. We found that LUADT1 was downregulated in sepsis and might regulate the miR-195/Pim-1 axis to inhibit LPS-induced of HCAEC apoptosis. This observation is of great significance for the clinical treatment of patients with sepsis (Fig. 5).

Heart is one of the most commonly affected organs by sepsis, and sepsis-induced heart failure leads to unacceptable high mortality rate. Sepsis-induced cardiomyopathy (SIC) is an infection-related cardiovascular disease. Studies have shown that at least $50 \%$ of patients with septic shock are clinically diagnosed with septic cardiomyopathy (Condor et al. 2017; Hong et al. 2018). Therefore, properly handling with the sepsis-induced heart failure became the urgent goal for all physicians worldwide. Previous studies have revealed that the apoptosis of cardiomyocyte and cardiac endothelial cells is involved in the pathological mechanism of cardiomyopathy (Uchida et al. 2010; Steiner and Lang 2017). In our study, overexpression of LUADT1 decreased the apoptotic rate of HCAECs under the treatment of LPS. Thus, LUADT1 may serve as a potential therapeutic drug for protecting patients' hearts through the treatment of sepsis. However, animal model and clinical studies are needed to further verify the clinical applications of LUADT1. In addition, the protective effects of LUADT1 on other organs remain to be further elucidated.

The development and progression of sepsis is accompanied by changes in the expression of multiple miRNAs (Vasilescu et al. 2017). Some of the differentially expressed miRNAs have been reported to play

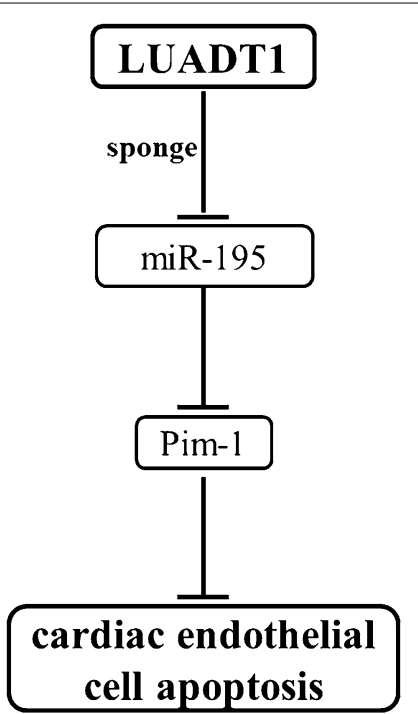

Fig. 5 Schematic figure outlining the mechanism by which LUADT1 protects cardiac endothelial cells against LPS-induced apoptosis critical roles in sepsis (Ge et al. 2018; Wu et al. 2018). For instance, overexpression of miR-214 improved cardiac function, reduced inflammation and myocardial injury (Ge et al. 2018). In contrast, overexpression of miR-223 is significantly correlated with inflammatory responses and poor prognosis of sepsis patients (Wu et al. 2018). In a recent study, miR-195 was overexpressed in mouse sepsis model, and downregulation of miR-195 inhibited injuries and cell apoptosis in multiple organs (Zheng et al. 2015). Consistently, our study further confirmed the role of miR-195 in promoting the apoptosis of HCAECs under the treatment LPS. Herein, miR-195 and LUADT1 were predicted to interact with each other. Although miR195 and LUADT1 did not affect the expression of each other, overexpression of LUADT1 led to the upregulation of Pim-1, a target of miR-195. Pim-1 was reported to regulate cardiomyocyte survival and promote the proliferation of cardiac progenitor cells, which provides a perspective in combining LUADT1 and Pim-1 in heart protection (Muraski et al. 2007; Cottage et al. 2012). Our data supported the idea that LUADT1 might sponge miR-195 to upregulate Pim-1.

\section{Conclusions}

In conclusion, LUADT1 is downregulated in sepsis and may regulate the miR-195/Pim-1 axis to inhibit the apoptosis of HCAECs induced by LPS.

\section{Abbreviation}

ncRNAs: Non-coding RNAs.

\section{Acknowledgements}

Not applicable

\section{Authors' contributions}

Study concepts: L-HW, study design: Z-MZ, definition of intellectual content: Z-MZ, literature research: M-ZL, clinical studies: Z-MZ, experimental studies: ZZ, data acquisition: XW, data analysis: XW, statistical analysis: M-ZL, manuscript preparation: D-LJ, manuscript editing: D-LJ, manuscript review: D-LJ. All authors read and approved the final manuscript.

\section{Funding}

Not applicable.

\section{Availability of data and materials}

The analyzed data sets generated during the study are available from the corresponding author on reasonable request.

\section{Ethics approval and consent to participate}

The present study was approved by the Ethics Committee of Affiliated Dongfeng Hospital, Hubei University of Medicine. The research has been carried out in accordance with the World Medical Association Declaration of Helsinki. All patients and healthy volunteers provided written informed consent prior to their inclusion within the study.

Consent for publication

Not applicable.

Competing interests

The authors declare they have no conflict of interests. 


\section{Author details}

1 Department of Critical Care Medicine, Affliated Dongfeng Hospital, Hubei University of Medicine, Shiyan 442008, Hubei, People's Republic of China.

2 Department of Clinical Laboratory, Affliated Dongfeng Hospital, Hubei University of Medicine, Shiyan 442008, Hubei, People's Republic of China.

${ }^{3}$ Department of Children's Medical Center, Affliated Dongfeng Hospital, Hube University of Medicine, Shiyan 442008, Hubei, People's Republic of China.

Received: 17 March 2020 Accepted: 13 October 2020

Published online: 23 November 2020

\section{References}

Cohen J, Vincent JL, Adhikari NKJ, et al. Sepsis: a roadmap for future research. Lancet Infect Dis. 2015;15(5):581-614.

Condor JM, Rodrigues C, Moreira R, et al. Human Wharton's jelly-derived mesenchymal stem cells improve sepsis-associated heart and lung injury. Cytotherapy. 2017;19(5):S197.

Cottage $C T$, Neidig L, Sundararaman B, et al. Increased mitotic rate coincident with transient telomere lengthening resulting from pim-1 overexpression in cardiac progenitor cells. Stem Cells. 2012;30(11):2512-22.

Cronin L, Cook DJ, Carlet J, et al. Corticosteroid treatment for sepsis: a critical appraisal and meta-analysis of the literature. Crit Care Med. 1995;23(8):1430-9.

Flores C. Host genetics shapes adult sepsis survival. Lancet Respir Med. 2015;3(1):7-8

Ge C, Liu J, Dong S. MIRNA-214 protects sepsis-induced myocardial injury. Shock. 2018;50(1):112-8.

Giamarellos-Bourboulis EJ, Opal SM. The role of genetics and antibodies in sepsis. Ann Transl Med. 2016:4(17):328.

Gotts JE, Matthay MA. Sepsis: pathophysiology and clinical management. BMJ. 2016:353:11585.

Hong G, Zheng D, Zhang L, et al. Administration of nicotinamide riboside prevents oxidative stress and organ injury in sepsis. Free Radic Biol Med. 2018;123:125-37.
Hotchkiss RS, Karl IE. The pathophysiology and treatment of sepsis. N Engl J Med. 2003;348(2):138-50.

Liu VX, Fielding-Singh V, Greene JD, et al. The timing of early antibiotics and hospital mortality in sepsis. Am J Respir Crit Care Med. 2017;196(7):856-63.

Muraski JA, Rota M, Misao Y, et al. Pim-1 regulates cardiomyocyte survival downstream of Akt. Nat Med. 2007:13(12):1467-75.

Qiu M, Xu Y, Wang J, et al. A novel IncRNA, LUADT1, promotes lung adenocarcinoma proliferation via the epigenetic suppression of p27. Cell Death Dis. 2015;6(8):e1858.

Riedemann NC, Guo RF, Ward PA. Novel strategies for the treatment of sepsis. Nat Med. 2003;9(5):517-24.

Steiner JL, Lang CH. Etiology of alcoholic cardiomyopathy: mitochondria, oxidative stress and apoptosis. Int J Biochem Cell B. 2017:89:125-35.

Uchida Y, Egami H, Uchida Y, et al. Possible participation of endothelial cell apoptosis of coronary microvessels in the genesis of Takotsubo cardiomyopathy. Clin cardiol. 2010;33(6):371-7.

van der Poll T, van de Veerdonk FL, Scicluna BP, et al. The immunopathology of sepsis and potential therapeutic targets. Nat Rev Immunol. 2017;17(7):407-20.

Vasilescu C, Dragomir M, Tanase M, et al. Circulating miRNAs in sepsis-A network under attack: An in-silico prediction of the potential existence of miRNA sponges in sepsis. PLoS ONE. 2017;12(8):e0183334.

Wu X, Yang J, Yu L, et al. Plasma miRNA-223 correlates with risk, inflammatory markers as well as prognosis in sepsis patients. Medicine. 2018;97(27):e11352.

Zheng D, Yu Y, Li M, et al. Inhibition of microRNA 195 prevents apoptosis and multiple-organ injury in mouse models of sepsis. J Infect Dis. 2015;213(10):1661-70.

\section{Publisher's Note}

Springer Nature remains neutral with regard to jurisdictional claims in published maps and institutional affiliations.
Ready to submit your research? Choose BMC and benefit from

- fast, convenient online submission

- thorough peer review by experienced researchers in your field

- rapid publication on acceptance

- support for research data, including large and complex data types

- gold Open Access which fosters wider collaboration and increased citations

- maximum visibility for your research: over 100M website views per year

At BMC, research is always in progress.

Learn more biomedcentral.com/submissions 